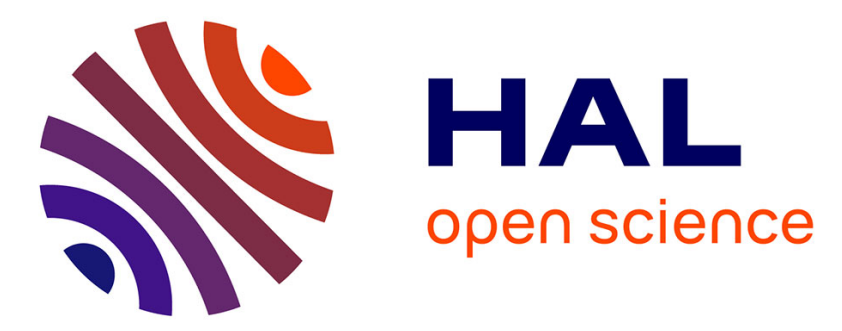

\title{
How to correctly assess mortality benefits in public policies
}

\author{
Olivier Chanel, Pascale Scapecchi, Jean-Christophe Vergnaud
}

\section{To cite this version:}

Olivier Chanel, Pascale Scapecchi, Jean-Christophe Vergnaud. How to correctly assess mortality benefits in public policies. Journal of Environmental Planning and Management, 2006, 49 (5), pp.759 - 776. 10.1080/09640560600850150 . halshs-00150092

\section{HAL Id: halshs-00150092 \\ https://shs.hal.science/halshs-00150092}

Submitted on 30 May 2007

HAL is a multi-disciplinary open access archive for the deposit and dissemination of scientific research documents, whether they are published or not. The documents may come from teaching and research institutions in France or abroad, or from public or private research centers.
L'archive ouverte pluridisciplinaire HAL, est destinée au dépôt et à la diffusion de documents scientifiques de niveau recherche, publiés ou non, émanant des établissements d'enseignement et de recherche français ou étrangers, des laboratoires publics ou privés. 


\title{
How to correctly assess mortality benefits in public policies
}

\author{
Olivier Chanel \\ CNRS-GREQAM-IDEP, 2 rue de la Charité, F-13002 Marseille, France \\ Pascale Scapecchi \\ OECD, 2 rue André Pascal, F-75016 Paris, France \\ and

\section{Jean-Christophe Vergnaud} \\ CNRS-EUREQua, 106-112 bd de l'Hôpital, F-75013 Paris, France
}

Corresponding author: Olivier Chanel, GREQAM, 2 Rue de la Charité, 13002 Marseille, France, Tel.: + 33491140 780; fax: + 33491900 227; e-mail: chanel@univmed.fr

The authors are very grateful to Alan Kirman, Stéphane Luchini, Ari Rabl, Lise Rochaix, Marjorie Sweetko and two anonymous referees for their helpful suggestions. 


\begin{abstract}
This paper concerns the difficulty of taking long-term effects on health into account in an economic valuation. Indeed, public decision-makers should incorporate the cessation lag between implementation of an abatement policy and achievement of all of the expected mortality-related benefits for any projects involving health impacts. We show how this time-lag problem can be handled by proposing two approaches - either in terms of deaths avoided or of life years saved - within a dynamic perspective. The main findings are that long-term health benefits calculated by standard methods and widely applied to adverse health effects should be corrected downwards when incorporated into an economic analysis. The magnitude of correction depends on the discount rate, on technical choices dealing with epidemiology and on the method chosen to assess mortality benefits.
\end{abstract}

\title{
INTRODUCTION
}

Improvements in data collection, accumulation of epidemiological studies and an increased concern for public health have resulted in better knowledge of long-term human health outcomes resulting from past exposure to adverse factors. Studies on the effects on health of environmental and occupational exposure to adverse substances have generally shown that long-term effects on health (i.e. chronic health outcomes resulting from long-term exposure) are much more severe than short-term ones (i.e. acute health outcomes immediately following exposure). They account for almost all the health benefits of giving up smoking and more than $70 \%$ of the health benefits of air pollution abatement (Ostro \& Chestnut, 1998; Holland \& King, 1999; Sommer et al., 1999). The assessment of future health benefits should therefore incorporate long-term effects on health as a prerequisite for evaluating the desirability of a public policy.

Long-term effects are characterized by the substantial time that elapses between the implementation of a regulation and the achievement of full health benefits. In the recent U.S. literature (see US EPA 2001, 2004), this issue is 
referred to as "cessation lag". We adopt this terminology to characterize the timelag between the reduction in exposure and the decrease in the health risk considered. Since chronic effects result from cumulative exposure, health expenditures observed over a given year do not depend solely on exposure to adverse substances in that year alone. This clearly implies that a decrease or an abatement in exposure will not fully and immediately reduce the associated health expenditures, but rather that there will be a time-lag before this is achieved. Failure to adequately take into account the lag factor may lead to incorrect assessment of future benefits. Ignoring the specific nature of long-term effects is equivalent to considering that all the benefits will accrue immediately after implementation of a policy, and, as a result, will lead to their overestimation. This has major consequences for economic valuation and therefore for public decisionmaking, particularly when discount rates are high, and it could be an important issue in certain health policies.

Several studies have recently assessed long-term effects on health by multiplying the number of attributable cases by the appropriate monetary values (see for instance Ostro \& Chestnut, 1998; Sommer et al., 1999 for air pollution exposure, or US EPA, 2000 for arsenic in drinking water). ${ }^{1}$ Most studies do not mention that the results represent benefits that can be obtained in the long run. Problems arise when the long-term nature of the underlying health outcomes is neglected and the overestimated benefits are compared with the correctly estimated costs of a policy, thereby biasing the analysis. The degree of overestimation of these benefits is of particular interest.

This paper proposes a methodology that takes into account the cessation lag associated with long-term effects on mortality. The methodology applies to various public health policies with lagged health effects, such as reductions in exposure to air pollution, chemicals or harmful radiation, campaigns for exercise, diet, healthier food or vaccination, or against alcoholism or smoking. It assesses both the number of deaths avoided and the years of life saved during the implementation of a health policy in a discrete time dynamic perspective. We depart from Leksell \& Rabl (2001) who propose a continuous time method based 
on years of life saved. We perform a sensitivity analysis with respect to various parameters, especially the magnitude of the cessation lag and the value of the discount rate - both of which are subject to severe uncertainty.

The paper proceeds as follows. Section 2 shows how the reduction in exposure to an adverse factor affects mortality. Section 3 presents a general framework for health benefits assessment when there are long-term effects. Section 4 provides conclusions.

\section{HOW REDUCTION IN EXPOSURE TO AN ADVERSE FACTOR AFFECTS MORTALITY}

Consider a decision-maker who wants to implement a regulation or an abatement policy that would generate short-term and long-term health benefits by improving the health of the population. In order to estimate the health benefits arising from the policy, one has first to estimate the health outcomes by combining epidemiological data with initial exposure level and exposure reduction. In this section, we show how to carry out this evaluation and introduce the issue of effects on mortality.

\section{The concepts of relative risk and death rates}

The concept of Relative Risk $(R R)$ is crucial in epidemiology, and is the starting point of the analysis. It can be defined as the risk, for a population exposed to a specific factor, of being affected by an event $\left(R_{E}\right)$, divided by the same risk for a population not exposed to this factor $\left(R_{N E}\right)$. This concept applies for both shortterm effects on health (within hours or days after exposure) and long-term effects on health (over years or even a lifetime). It should be noted that the population is heterogeneous, hence the $R R$ may vary within the population. We consider here that an average $R R$ can be defined for a given health indicator depending on the 
current average level of exposure. $R R_{E} \equiv \frac{R_{E}}{R_{N E}}$ denotes the relative risk for a level of exposure $E$.

As the level of exposure changes due to the implementation of a regulation or an abatement policy, the $R R$ varies according to two key variables: the length of the time-lag between past (long-term) exposure and its future health consequences, and, for some illnesses, the way the human body heals itself after a period of lower exposure. Thus, we can assume that the $R R$ of a health indicator follows a declining pattern:

$$
R R(t)=g(E, \Delta, t)
$$

where

- $R R(t)$ denotes the relative risk $t$ years after reduction,

- $E$ is the initial level of exposure,

- $\Delta$ stands for the fractional exposure reduction $(\Delta \in[0,1])$,

- $g$ is a functional form, with $\partial g / \partial t<0, \partial g / \partial E>0$ and $\partial g / \partial \Delta<0$.

It should be noted that at the date of reduction $t=0, R R(0) \equiv R R_{E}$ and that $R R(t)$ approaches $R R_{(1-\Delta) E} \equiv \frac{R_{(1-\Delta) E}}{R_{N E}}$ as $t \rightarrow \infty$.

This paper deals with mortality risks only. Hence, reduction in exposure to an adverse factor affects mortality rates by modifying the relative risk of death. Let $D(0 ; x)$ be the mortality rate observed at age $x$ before the reduction, composed of one part affected by the reduction and another part independent of the reduction:

$$
D(0 ; x)=D^{R}(0 ; x)+D^{U}(0 ; x)
$$

where

- $D^{R}(0 ; x)$ is the death rate at age $x$ for causes directly linked to the factor in question,

- $D^{U}(0 ; x)$ is the death rate at age $x$ for unrelated causes. 
To make this distinction clearer, it should be remembered that accidental deaths, for instance, are not affected by a reduction in exposure to chemicals, nor are deaths due to occupationally-induced cancers affected by safety improvements in road infrastructures. ${ }^{2}$

The relevant death rate $t$ years after implementation of the policy varies according to $R R(t)$ :

$$
D^{R}(t ; x)=\frac{R R(t)}{R R(0)} D^{R}(0 ; x)
$$

It will vary from $D^{R}(0 ; x)$ when $t=0$ to $\frac{R R_{(1-\Delta) E}}{R R(0)} D^{R}(0 ; x)$ when $t \rightarrow \infty$.

By definition, deaths due to unrelated causes are considered not to be affected by the health factor: $D^{U}(t ; x) \equiv D^{U}(0 ; x)$ for all $t$. Hence, the total death rate at age $x$ and $t$ years after the reduction is:

$$
D(t ; x)=D^{R}(t ; x)+D^{U}(0 ; x)
$$

\section{Instantaneous and complete removal of risk}

To characterize accurately the way $R R(t)$ varies, i.e. the shape of function $g$, requires extensive information and a lengthy observation period, which explains the scarcity of available epidemiological data. A noteworthy exception is the case of smoking cessation, for which many studies indicate that mortality among former smokers approaches the level of lifelong non-smokers after a smoking cessation time of 10 to 20 years (see for instance Kawachi et al, 1993; Doll et al., 1994 or Leffondré et al., 2002). When studying emissions from non-road diesel engines, the US EPA (2004) suggests that $20 \%$ of the mortality reductions may occur in the first year, 50\% may be spread equally over the next four years, and the remaining $30 \%$ may be spread equally over the next 15 years.

We present a simple general approach to characterize $g$ before considering possible extensions. Lightwood \& Glantz (1997) estimate a mortality risk function based on the meta analysis of seven studies of giving up smoking, an impact 
characterized by instantaneous and complete risk removal. Eq. (5) is derived from their risk function, and presents a general equation for such an impact:

$$
R R(t)=\left(R R_{E}-R R_{N E}\right) \times \exp \left(-\frac{t}{\tau}\right)
$$

where

- $t$ is the time in years since the exposure was stopped,

- $R R_{N E}$ denotes the relative risk of an impact-related illness for those not exposed to the impact (by definition $R R_{N E}=I$ ),

- $R R_{E} \equiv R R(0)$ denotes the relative risk of an impact-related illness before the impact ceases $(t=0)$,

- $\tau$ is the time constant (in years) of the exponential function, assumed to be illness-dependent.

The estimates of $\tau$ differ in the literature depending on the illness considered. Lightwood \& Glantz (1997) obtain 1.4 for stroke and 1.6 for acute myocardial infarction, Leksell (2000) suggests between 4.3 and 6.5 for lung cancer, and Doll et al. (1994) between 10 and 15 for total excess risk. The negative exponential function in Eq. (5) is also found to fit adequately decay phenomena in other disciplines (e.g. physics, biology...), and is hereunder considered as benchmark. ${ }^{3}$ However, the direct transposition of the smoking cessation results to other contexts raises difficulties due to differences in agents (gas vs. particulate matter), biokinetics, bioaccumulation, exposure pathways, target organs, magnitude of the reduction in exposure.... (see for instance the debate on applying smoking/lung cancer functions to arsenic/bladder cancer, US EPA, 2001). Epidemiological data specific to the impact-related illness at stake should thus be favoured and, at the least, a sensitivity analysis on the value of $\tau$ should be carried out.

Figure 1 indicates how the relative risk $R R(t)$ decreases with time according to Eq. (5), moving from $R R_{E}$ toward the $R R$ of non-exposed subjects $(R R=1)$. The results depend strongly on the value of $\tau$, as it takes 7 years to reach $R R=1$ when $\tau=1$, but up to 45 years when $\tau=10$. 
[Figure 1 about here].

It should be noted that epidemiological studies compute the $R R_{E}$ on subjects exposed to the adverse factor under consideration before the impact ceases. Hence, Eq. (5) should only apply to this population, exposed for at least as long as required for the $R R$ to reach the equilibrium (30 years is a reasonable value according to Figure 1). ${ }^{4}$ The present framework is consequently not appropriate to study child mortality, for instance.

Although adverse effects on health related to tobacco smoke are only longterm effects, adverse effects on health are in general a mix of short-term and longterm effects. Short-term effects will disappear as soon as the exposure to the risk factor ceases while long-term effects will evolve gradually. Therefore, let us split $R R_{E}-R R_{N E}$ into two parts, with $R_{E}^{S T}$ denoting the short-term effects (i.e. less than one year) share and $R_{E}^{L T}$ the long-term effects (i.e. more than one year) share. In order to keep the formulation as general as possible, we choose an exponential decrease for both effects and Eq. (5) becomes:

$$
R R(t)=R R_{N E}+\sum_{i=S T, L T} R_{E}^{i} \times \exp \left(-\frac{t}{\tau^{i}}\right)
$$

Note that all short-term effects virtually disappear within one year once $\tau^{S T} \leq 0.1$

\section{Extension to a non-instantaneous and incomplete removal of risk}

For many risk factors, abatement policy constraints or technical constraints preclude instantaneous and complete exposure reduction. Examples of such policies are the introduction of filters that reduce industrial and car emissions, thorough vaccination campaigns, alcohol or tobacco prevention policies, regulations concerning exposure to toxic substances... Thus, we consider a gradual policy of duration $p$, i.e. that takes $p$ years to achieve a fractional percentage reduction $\Delta \in[0,1]$. Below, we consider the simplest case of a linearly decreasing reduction: each year, an additional reduction of $\Delta / p$ occurs. We consequently have 
to generalize Eq. (6) in two ways.

First, if we consider an incomplete reduction $\Delta<1$, the relative risk will approach $\mathrm{RR}_{(1-\Delta) \mathrm{E}}$ in the long run following the negative exponential path:

$$
R R(t)=R R_{(1-\Delta) E}+\sum_{i=S T, L T} R_{(1-\Delta) E}^{i} \times \exp \left(-\frac{t}{\tau^{i}}\right)
$$

Second, removal of the exposure is no longer considered instantaneous. It is supposed that a reduction of $\Delta$ will be achieved over $p$ years following a linearly decreasing path $(\Delta / p$ every year $t \leq p)$. The impact on the $R R$ will be proportional to the decline during the $p$ first years, and will fully apply after $p$ years:

when $t=0, R R(0)=R R_{(1-\Delta) E}+\sum_{i=S T, L T} R_{(1-\Delta) E}^{i} \times \exp \left(-\frac{t}{\tau^{i}}\right)$

when $t=1, R R(1)=R R_{(1-\Delta) E}+\sum_{i=S T, L T} \frac{R_{(1-\Delta) E}^{i}}{p}\left[(p-1)+\exp \left(-\frac{t}{\tau^{i}}\right)\right]$

when $t \leq p, R R(t)=R R_{(1-\Delta) E}+\sum_{i=S T, L T} \frac{R_{(1-\Delta) E}^{i}}{p}\left[(p-t)+\sum_{h=1}^{t} \exp \left(-\frac{h}{\tau^{i}}\right)\right]$

when $t \geq p, R R(t)=R R_{(1-\Delta) E}+\sum_{i=S T, L T}\left[\frac{R_{(1-\Delta) E}^{i}}{p} \sum_{h=t-p+1}^{t} \exp \left(-\frac{h}{\tau^{i}}\right)\right]$

where $h$ is a time indicator.

The general formulation becomes:

$$
R R(t)=R R_{(1-\Delta) E}+\sum_{i=S T, L T}\left[\frac{R_{(1-\Delta) E}^{i}}{p} \sum_{h=t-p+1}^{t} \exp \left(-\frac{\operatorname{Max}(h, 0)}{\tau^{i}}\right)\right]
$$

This expression can be generalized further by choosing as many terms $i$ as required to account for effects on health of different time constants $\tau^{i}$. Figure 2 represents the evolution of $R R(t)$ for $\tau^{S T}=0.1$ and $\tau^{L T}=5, R_{E}^{S T}$ standing for $25 \%$ of total excess risk, and different values for $p$. For instance, the excess relative risk is divided by two after 2 years for $p=1$, whereas it takes 13 years to obtain the same 
reduction if $p=20$.

[Figure 2 about here].

This affects the rates of incidence of the relevant health indicators and therefore the number of years necessary to reap full health benefits from a reduction policy. We need to transform changes in death rates into a health indicator, and then into a monetary value. This is done in the next section, which presents a framework specific to the problem at hand.

\section{INCLUSION OF LONG-TERM EFFECTS WITHIN AN ECONOMIC ASSESSMENT}

In order to assess whether it is economically efficient to implement a given public policy, its benefits must be compared to its costs. A cost-benefit analysis generally compares the future discounted costs and the benefits of a policy (see Gramlich, 1990 or Layard \& Glaister, 1994 for a general overview). Although reduction in the level of exposure generates health improvements both in terms of mortality and morbidity, in this paper we are only interested in the challenge of properly assessing the benefits with respect to mortality.

\section{Defining the problem of measuring decrease in mortality}

Insofar as our aim is to take into account the "cessation lag", a dynamic setting is required. Two approaches are possible.

The first approach consists in focusing on the number of deaths avoided, a common concept in environmental economics. It raises certain issues which need to be dealt with.

First, to assess the benefits in terms of number of deaths avoided is - in some cases- not appropriate, since this number cannot always be determined correctly from epidemiological studies (see for instance Rabl, 2003, for cardio-pulmonary mortality due to air pollution exposure). 
This is further complicated by the fact that deaths attributable to an adverse effect on health are often assessed by considering the difference between the number of deaths observed in a population exposed to a given level of adverse environmental factors and the number of deaths that would occur in a non-exposed population. ${ }^{5}$ This is incorrect when long-term effects are involved for two reasons. First, the cessation lag factor is ignored although the decrease in $R R_{E}$ will not immediately follow risk removal, but will occur progressively (see the general formulation of $R R(t)$ in Eq. (8)). Second, deaths avoided in a given year will inevitably occur in the future!

The problem of the cessation lag cannot be solved easily just by extending calculations of the difference in number of deaths in a dynamic setting. Indeed, consider a hypothetical population - initially in a steady state according to initial death rates observed in current mortality tables - which evolves according to the relative risk in Eq. (8). The annual number of deaths will first decrease as a consequence of the reduction of $R R(t)$. Since these avoided deaths are simply postponed for the future, the population will reach a new steady state in the long run, where the annual number of deaths is the same as initially! Figure 3 shows how the number of deaths avoided evolves, for both instantaneous and complete risk removal and for immediate decrease in $R R$ (this case is referred to as $\tau^{\mathrm{LT}}$ in the sequel) in a population. When $\tau^{L T}=5$, it takes 7 years to reach $50 \%$ of the annual maximum number of deaths avoided when $\tau^{L T}=0$ (the maximum is reached in the first year in this case). In both cases, the number of deaths avoided slowly decreases towards 0 , which is reached about 60 years after the beginning of the policy.

[Figure 3 about here].

The number of deaths avoided the first year in the case $\tau^{L T}=0$ (see Figure 3 ) is the measure actually used in the literature, but it ignores cessation lags. The benefits of a permanent policy are then (wrongly) computed by considering the flow of deaths avoided on this basis. The question of how to correctly count the number of deaths avoided in a dynamic setting and how to incorporate the 
cessation lag clearly deserves particular attention.

The second approach is based on the number of years of life saved, a widely-used concept in health economics (see Johansson, 1995 or Mehrez and Gafni, 1989). Thus, every year, the total number of years lived by the population (i.e. the total number of persons alive) is computed, as well as the gains in life expectancy. The total discounted number of life years saved by a given policy is easier to derive as shown in the next sections.

\section{Correctly accounting for a decrease in mortality}

When assessing the effect of a population's decrease in mortality, the variation process is as follows.

- The population is initially in steady state, with $N(t ; 0) \equiv N$ the number of births in year $t$, assumed to be constant. The number of persons of age $x$ alive at date $0, N(0 ; x)$, is computed from the product of all the survival rates before age $x: \quad N(0 ; x)=\prod_{t=0}^{x-1}[1-D(0 ; y)] N$. The number of years lived in year 0 is $L(0 ;)=.\sum_{x} N(0 ; x)$.

- The number of persons of age $x$ alive at date $t$ is computed from the number of people of age $x-1$ alive at date $t-1$, which is affected by the survival rate of people of age $x-1$ at date $t-1: \forall x \geq 1, \forall t \geq 1, N(t ; x)=[1-D(t ; x-1)] N(t-1 ; x-$ 1), with $D(t ;$.) evolving as defined in the second section.

- The number of deaths avoided at age $x$ in year $t$ is $N(t ; x)[D(0 ; x)-D(t ; x)]$ and the total number of years lived in year $t$ is $L(t ;)=.\sum_{x} N(t ; x)$.

The number of life years saved (NLYS) in year $t$ by the policy is $L(t ;)-.L(0 ;$.$) ,$ while the gain in terms of life expectancy at birth is obtained by dividing this number by $N$. The number of policy-related deaths avoided (NPDA) in year $t$ is $\sum_{x} N(t ; x)[D(0 ; x)-D(t ; x)]$. The NPDA is a relevant concept since the deaths 
avoided by the policy in a given year remain in the population and must be assumed to be exposed to other competing mortality risk factors: one way or another, they will be counted as deaths (remember Figure 3). Both indicators increase until the population reaches another steady state corresponding to $R R(t)=R R_{(1-\Delta) E}$. In a given year $t$, they depend on the initial level of exposure $E$, and the interaction of three parameters: the level of reduction $(\Delta)$, the length of time until the policy is fully implemented $(p)$ and the parameters of the risk function $\left(\tau^{i}\right)$.

\section{Sensitivity of the decrease in mortality to the parameters}

Let us arbitrarily set the share of the short-term effects $R_{E}^{S T}$ at $25 \%$ of the total excess risk in the remainder of the paper, as in Figure 2. Consider first the influence of $\tau^{L T}$ and $p$ on the number of deaths avoided. Figure 4 represents the time necessary to obtain the full effects for $\tau^{L T}=5$ and different values of $p$ (the time lapse also depends on $R R_{E}$, but so slightly that it does not show up in the Figure). French mortality data were used to characterize the initial steady state. ${ }^{6}$

[Figure 4 about here]

If the reduction is complete and instantaneous $(p=1)$, it takes one year $\left(\tau^{L T}=1\right)$ to seven years $\left(\tau^{L T}=10\right)$ to obtain $50 \%$ of the maximum effect. If $p=20$ years, the number of years is respectively 11 and 18 . Thus it appears that when $\tau^{L T}=10$ instead of $\tau^{L T}=1$, it takes 7 more years to reach half the overall benefits, and 30 more years to reach $99 \%$ of the overall benefits. When the term of the policy is $p=20$ (years) instead of $p=1$, it takes about 11 more years to reach fifty percent of the overall benefits.

Similar results are obtained with the approach in terms of number of life years saved but the time necessary to obtain the full benefits is longer. This is quite normal since a death avoided at date $t$ counts as a health benefit at date $t$ in the NPDA approach whereas in the NLYS approach, the years of life saved corresponding to the postponed death spread over the expected remaining life 
expectancy at date $t$, i.e. at date $t, t+1, t+2, \ldots$

Thus, economic consequences will be substantial, especially when discount rates are high, since the computations must then take into account time lapses of up to 30 years before including the entire benefits. Ignoring these time-lags leads to an overestimation of the total discounted number of deaths avoided.

\section{Assessing the consequences of ignoring the cessation lag}

Let us consider how cessation lags affect the total discounted NPDA and NLYS, and the consequences of ignoring them.

The total discounted number of policy-related deaths avoided is:

$$
\operatorname{TNPDA}\left(\delta, R R_{E}, \tau^{L T}, p, \Delta\right)=\sum_{t=0}^{\infty} \frac{1}{(1+\delta)^{t}} N P D A\left(R R_{E}, \tau^{L T}, p, \Delta, t\right)
$$

where $\delta$ denotes the annual discount rate. Discounting reflects the interaction of temporal preference relative to deaths avoided at different dates and the opportunity cost of economic resources devoted to the public health policy. The market interest rate is generally considered as a valid approximation and $\delta$ is the subject of a sensitivity analysis hereunder. ${ }^{7}$

When the cessation lag is ignored (i.e. $\tau^{L T}=0$ ), the total discounted number of policy-related deaths avoided is noted $\operatorname{TNPDA}\left(\delta, R R_{E}, 0, p, \Delta\right)$. Clearly, $T N P D A\left(\delta, R R_{E}, 0, p, \Delta\right) \quad$ exceeds $T N P D A\left(\delta, R R_{E}, \tau^{L T}, p, \Delta\right)$ for $\tau^{L T}>0$. The importance of the cessation lag can be obtained by considering the ratio $C \equiv \frac{T N P D A\left(\delta, R R_{E}, \tau^{L T}, p, \Delta\right)}{T N P D A\left(\delta, R R_{E}, 0, p, \Delta\right)}$ that needs to be applied to $T N P D A\left(\delta, R R_{E}, 0, p, \Delta\right)$ to get the correct value.

Formally, $C$ depends on five parameters: $\delta, R R_{E}, \tau^{L T}, p$ and $\Delta$ and simulations have been made taking different values for these parameters. Since no specific risk factor has been selected, we can consider that the policy-maker aims to reduce the relative risk from $R R_{E}$ to $R R=1$. We have considered a large range of values for the parameters: 
$-\delta$ varies from 0.01 to 0.08 with a step size of 0.01 ,

$-R R_{E}$ covers the range from 1 to 1.5 with a step size of 0.05 ,

$-\tau^{L T}$ varies between 1 and 10 ,

$-p=1,5,10,20$ years,

$-\Delta$ varies from 0.05 to 1 with a step size of 0.05 .

For a given policy, $\tau^{L T}$ and $\delta$ have the strongest impact on $C$ whereas the impact of $R R_{E}$ is weak. $C$ is plotted in Figure 5 for different values of $\delta$ and $\tau^{L T}$ in the case of a ten-year policy achieving a full risk reduction $(p=10$ and $\Delta=1)$.

[Figure 5 about here].

$C$ is found to lie between 0.45 and 0.93 , with a value around 0.71 when $\tau^{L T}=5$ and $\delta=0.04$. The impact of each of the parameters on $C$ is negative. Indeed, the lower $\tau^{L T}$, the higher $C$, which could be explained by the fact that small values of $\tau^{L T}$ imply a rapid decrease in $R R$ following the implementation of the reduction policy. The smaller the discount rate, the higher $C$ due to classical discounting effect. Lastly, the lower $R R_{E}, p$ or $\Delta$, the higher $C$. Indeed, the impact of ignoring the cessation lag lessens as the risk-reduction policy becomes quicker, more partial and more related to a low $R R$.

The total discounted number of life years saved is computed like the TNPDA (see Eq. (9)) and the ratio $C$ computed as well. Similar impacts of the parameters on $C$ are obtained. Note however that the longer time required to obtain the full benefits (see above) implies a lower $C$ due to higher discounting effect. Indeed, $C$ is found to lie between 0.27 and 0.85 , with a value around 0.53 when $\tau^{L T}=5$ and $\delta=0.04$.

[Figure 6 about here].

Whatever the approach, the conclusion is similar: the cessation lag has a potentially significant impact on the estimation of health benefits when not properly accounted for. 


\section{Economic valuation of health benefits}

The multitude of empirical assessments of a value for a prevented fatality (VPF) conducted so far have provided a large range of values (with a few exceptions between 0.7 and 6.1 million EUR). Such a large range should not be surprising, since there are major differences in methodology, in the attributes of the risk in question (whether or not it is controllable, familiar, dreadful, uncertain, voluntary, catastrophic, unfair, immediate, see Slovic, 1987) and in potential victim characteristics as well.

The cessation lag is rarely taken into account when monetizing benefits. When it is taken into account, this is done either by discounting the current VPF to reflect the time-lag or by deriving the VPF from willingness to pay estimates for future mortality risk reductions (Alberini et al., 2004). Neither of these methods is satisfactory, since they do not account for the cessation lag by relying on the epidemiological dynamic setting.

The methodology we propose can be adapted to any VPF, especially agedependent $V P F$, when the NPDA approach is used. Indeed, if the VPF at age $x$ is denoted by $\operatorname{VPF}(x)$, the total discounted benefits $B^{N P D A}($.$) associated to the$ reduction policy will be:

$$
B^{N P D A}\left(\delta, R R_{E}, \tau^{L T}, p, \Delta\right)=\sum_{t=0}^{\infty} \frac{1}{(1+\delta)^{t}}\left[\sum_{x=0}^{\infty} N(t ; x)[D(0 ; x)-D(t ; x)] V P F(x)\right]
$$

When the NLYS approach is used, a direct way to obtain a VOLY consists in assessing the willingness to pay to increase life expectancy by one year. Unfortunately, very few studies are available (see however Johannesson and Johansson, 1997 or Krupnick et al., 1999) and values strongly depend on the individual's age and the closeness of this extra year. An indirect way consists in deducing a VOLY from a VPF by considering the latter as a discounted sum of equally valued VOLYs (see for instance Viscusi et al., 1997 or Leksell et Rabl, 2001)

Whatever the way the VOLY is computed, the total discounted benefits $\mathrm{B}^{\mathrm{NLYS}}($.) will be: 


$$
B^{N L Y S}\left(\delta, R R_{E}, \tau^{L T}, p, \Delta\right)=\sum_{t=0}^{\infty} \frac{1}{(1+\delta)^{t}}\left[\sum_{x=0}^{\infty}[N(t ; x)-N(0 ; x)] \operatorname{VOLY}(x)\right]
$$

Once the approach and the corresponding relevant monetary value have been chosen, the proposed methodologies allow for a correct assessment of the benefits of a given policy, and its comparison to the corresponding costs.

\section{CONCLUSION}

More and more evaluations of effects on health lead to the conclusion that externalities are important, especially long-term ones which account for most of the overall effects. Thus, public decision-makers should incorporate them in costbenefit analyses for any project involving health impacts. The cessation lag issue we explore is found to be crucial from a decision-making standpoint. The purpose of the paper is methodological: we show how this problem can be handled and provide a framework which enables to estimate future benefit trends both in terms of number of policy-related deaths avoided and number of life years saved. To take into account the time-lag factor, we need to consider a dynamic perspective. In a cost-benefit analysis setting, benefits corresponding to long-term effects on health should then be corrected by a factor that is highly sensitive to the value chosen for the discount rate. Otherwise, consequences on public health may be dramatic, since a policy may generate a social loss rather than an expected social benefit.

Although only benefits linked to mortality have been explored here, longterm morbidity should also be studied. Unfortunately, very few epidemiological data exist for these effects on health, and their evaluation requires further research. The influence of long-term morbidity on the correction factor may well be surprising, since it largely postpones health costs for the future, which may appear desirable due to discounting. 


\section{Notes}

${ }^{1}$ The European Union followed this route exclusively until 1995, in particular within the ExternE framework. Since 1997, approaches using deaths avoided and years of life saved have been employed simultaneously.

${ }^{2}$ This is not absolutely true, since the medical resources freed by a decrease in one health problem might at least theoretically be used to treat another.

${ }^{3}$ Note for instance that the distribution of the mortality in the above-mentioned US EPA (2004) study more or less correspond to $\tau=3$ in Eq. (5).

${ }^{4}$ When past exposure to the adverse factor cannot be considered as constant, things are more complicated (see Leksell and Rabl, 2001, for an example dealing with air pollution exposure).

${ }^{5}$ See for instance Holland \& King (1999) and Olsthoorn et al. (1999) for the European Union, Ostro \& Chestnut (1998) and US EPA (2000) for the United States or Gynther \& Otterström (1998) for Finland.

${ }^{6}$ Death rates below 30 (see the remark above on past exposure) as well as accidental death rates at all ages have been considered as unrelated causes $\mathrm{D}^{\mathrm{U}}($.) in Eq. (2). Computations have been performed separately for males and females but have been pooled when discussing the results. Finally, data observed in OECD countries are very similar to French data and allow generalization of the following results to developed countries.

${ }^{7}$ See Gravelle \& Smith (2001) for a discussion on the way to discount health effects in cost-benefit and cost-effectiveness analyses. 


\section{REFERENCES}

Alberini, A., Cropper, M., Krupnick, A. \& Simon N. (2004) Willingness to pay for mortality risk reductions: Does latency matter?, NCEE Working Paper \# 0401.

Doll, R., Peton, R., Wheatley, K., Gray, R. \& Sutherland, I. (1994) Mortality in relation to smoking: 40 years' observations on male British doctors, British Medical Journal, 309, pp. 901-911.

Gramlich, E.M. (1990) A Guide to Benefit-Cost Analysis (New-Jersey: Prentice Hall).

Gravelle, H. \& Smith, D. (2001) Discounting for health effects in cost-benefit and cost-effectiveness analysis, Health Economics, 10, pp. 587-599.

Gynther, L. \& Otterström, T. (1998) Willingness to pay for better air quality including application to fuel conversion in buses from diesel to natural gas, TERA Conference 98, FEEM, Milan, Italy.

Holland, M. \& King K. (1999) Economic evaluation of a directive on national emission ceilings for certain atmosphere pollutants. Part B: Benefit Analysis, EU DGXII Report, November.

Johannesson, M. \& P.O. Johansson (1997) Quality of life and the WTP for an increased life expectancy at an advanced age, Journal of Public Economics, 65, pp. 219-228.

Johansson, P.O. (1995) Evaluating Health Risks, An Economic Approach. (Cambridge: Cambridge University Press).

Kawachi, I., Colditz, G., Stampfer, M., Willett, W., Manson, J., Rosner, B., Hunter, D., Hennekens, C. \& Speizer F. (1993) Smoking cessation in relation to total mortality rates in women: A prospective cohort study, Annals of Internal Medicine, 119(10), pp. 992-1000.

Krupnick, A., Alberini, A., Cropper, M., Simon, N., with Itaoka, K. \& M. Akai (1999) Mortality risk valuation for environmental policy, Discussion paper 
Resources for the Future \# 99-47.

Layard, R. \& Glaister, S. (1994) Cost-Benefit Analysis. (Cambridge: Cambridge University Press).

Leffondré, K., Abrahamowicz, M., Siemiatycki, J. \& Rachet B. (2002) Modeling smoking history: A comparison of different approaches, American Journal of Epidemiology, 156, pp. 813-823.

Leksell, I. (2000) Health costs of particle emissions - Economic valuation of increased mortality due to exhaust of fine particles, Göteborg University.

Leksell, I. \& Rabl, A. (2001) Air pollution and mortality: quantification and valuation of years of life lost, Risk Analysis, 21(5), pp. 843-857.

Lightwood, J.M. \& Glantz, S.A. (1997) Short-term economic and health benefits of smoking cessation, Circulation, 96, pp. 1089-1096.

Mehrez, A., \& Gafni A. (1989) Quality-adjusted life years, utility theory and healthy-years equivalent, Medical Decision Making, 9, pp. 142-149.

Olsthoorn, X., Amann, M., Bartoova, A., Clench-Aas, J., Cofala, J., Dorland, K., Guerreiro, C., Henriksen, J.Fr., Jansen, H. \& Larssen, S. (1999) Cost-benefit analysis of European air quality targets for sulfur dioxide, nitrogen dioxide and fine and suspended particulate matter in cities, Environmental and Resource Economics, 14, pp. 333-351.

Ostro, B. \& Chestnut, L. (1998) Assessing the health benefits of reducing particulate matter air pollution in the USA, Environmental Research section A, 76, pp. 94-106.

Rabl, A. (2003) Interpretation of air pollution mortality: number of deaths or years of life lost, Journal of the Air and Waste Management Association, 53(1), pp. 41-50.

Slovic, P. (1987) Perception of risk, Science, 236, pp. 280-285.

Sommer, H., Seethaler, R., Chanel, O., Herry, M., Masson, S. \& Vergnaud, J.C. (1999) Health costs due to road traffic-related air pollution, an impact 
assessment project of Austria, France and Switzerland, Economic valuation, WHO Technical Report TEH07.

U.S. Environmental Protection Agency (2000) Arsenic in drinking water rule economic analysis, EPA 815-R-00-026, December.

U.S. Environmental Protection Agency (2001) Arsenic rule benefits analysis: $A$ SAB review, EPA-SAB-EC-01-008.

U.S. Environmental Protection Agency (2004) Final regulatory impact analysis: control of emissions from nonroad diesel engines, EPA420-R-04-007.

Viscusi, K., Hakes, J. \& Carlin, A. (1997) Measure of mortality risks, The Journal of Risk and Uncertainty, 14 (3), pp. 213-233. 